\section{Alteraciones craneofaciales en niños y adolescentes obesos}

\author{
Craniofacial aterations in obese \\ children and adolescents
}

Alexandre Frascino ${ }^{1, a}$, Louise Cominato $1, \mathrm{~b}$

${ }^{1}$ Universidade de São Paulo, Instituto da Criança da Faculdade de Medicina. São Paulo, Brasil.

a Maestro en Cirugía Oral y Maxilofacial.

${ }^{\mathrm{b}}$ Maestra en Pediatría.

\section{Correspondencia:}

Alexandre Frascino

Correo electrónico: aledefra@gmail.com

Rua Pedro de Toledo, 108 - cj. 13 Vila Clementino - São Paulo SP - Brazil.

Fecha de recepción: 26/09/18

Fecha de aceptación: 02/10/18
El crecimiento y desarrollo craneofacial implica la interacción de factores locales y sistémicos, tales como la genética, la disponibilidad de nutrientes, las fuerzas musculares y parafunciones ${ }^{1}$. Aunque el papel de las alteraciones hormonales inherentes a la obesidad en la niñez y adolescencia sobre el crecimiento pondoestatural es bastante conocido, se ha estudiado poco los efectos de la obesidad sobre el crecimiento craneofacial.

La incidencia de obsesidad o sobrepeso de niños y adolescentes se ha incrementado en las últimas décadas. En los últimos diez años, un tercio de los niños norteamericanos fueron diagnosticados con sobrepeso y el 17\% por ciento como obesos. En América Latina, se observa el aumento del número de pacientes obesos en todas las edades y en ambos sexos. Uno de cada cinco niños se encuentra con sobrepeso o es considerado obeso ${ }^{2}$. Estas cifras revelan la importancia de profundizar los estudios referentes a las complicaciones asociadas a la obesidad, en especial al grupo de edad compuesto por los más jóvenes.

Diversas enfermedades crónicas se asocian a la obesidad, como enfermedades cardiovasculares, infarto agudo de miocardio, hipertensión arterial, hiperlipidemia, trombosis, enfermedades autoinmunes, enfermedades musculoesqueléticas, cambios de crecimiento y desarrollo, incluso el cáncer ${ }^{3}$.
Las alteraciones hormonales inherentes a la obesidad infantil pueden comprometer el crecimiento de todo el esqueleto ${ }^{4}$. Sin embargo, las estructuras de la cara responden a los estímulos hormonales de formas diferentes, presentando crecimiento desproporcional o aberrante. Se puede observar en estos pacientes, un aumento de la dimensión anteroposterior de la base del cráneo y de la mandíbula; prognatismo bimaxilar y crecimiento desproporcional de la mandíbula ${ }^{5}$. También se han descrito cambios en la cronología de la erupción en pacientes jóvenes obesos ${ }^{6}$.

Las alteraciones craneofaciales originadas en la infancia pueden comprometer la salud durante la fase adulta. Se ha reportado una mayor prevalencia de enfermedad periodontal y caries en pacientes obesos. Así mismo, existe una mayor prevalencia de enfermedades crónicas en adultos obesos, como enfermedades cardiovasculares y la diabetes, factores reconocidos y considerados de mal pronóstico para la salud oral ${ }^{7}$.

Para ayudar a combatir la obesidad entre niños y adolescentes, diversas políticas de salud pública han sido objeto de los esfuerzos gubernamentales y de las sociedades médicas. En el 2014, los países integrantes de la Organización Panamericana de la Salud (OPS) firmaron un acuerdo para desalentar el consumo de productos alimenticios industrializados con la adición de altas con- 
centraciones de azúcares, que resultó en una reducción del $11,6 \%$ del consumo de los mismos ${ }^{8}$.

Las principales causas relacionadas con el creciente número de niños y adolescentes obesos incluyen las alteraciones de hábitos de vida con disminución de actividad física, aumento de la disponibilidad para el consumo de alimentos con altos índices calóricos y en mayores proporciones, disminución de las horas de sueño y estrés 9 . Los cirujanos dentistas, junto con los demás profesionales de salud, tienen un rol importante, ya que deben participar del diagnóstico precoz y de la prevención de la obesidad en niños, adolescentes y adultos.

\section{Referencias bibliográficas}

1. Davidopoulou S, Chatzigianni A. Craniofacial morphology and dental maturity in children with reduced somatic growth of different aetiology and the effect of growth hormone treatment. Prog Orthod. 2017;18(1):10. doi: 10.1186/s40510-017-0164-2

2. Rtveladze K, Marsh T, Barquera S, Sanchez Romero LM, Levy D, Melendez G, et al. Obesity prevalence in Mexico: impact on health and economic burden. Public Health Nutr. 2014;17(1):233-9. doi: 10.1017/ S1368980013000086

3. Morrison JA, Friedman LA, Gray-McGuire C. Metabolic syndrome in childhood predicts adult cardiovascular disease 25 years later: the Princeton Lipid Research Clinics Follow-up Study. Pediatrics. 2007;120(2):340-5. doi: 10.1542/peds.2006-1699
4. Johnson W, Stovitz SD, Choh AC, Czerwinski SA, Towne B, Demerath EW. Patterns of linear growth and skeletal maturation from birth to 18 years of age in overweight young adults. Int J Obes (Lond). 2012;36(4):535-41. doi: 10.1038/ijo.2011.238

5. Olszewska K. Craniofacial morphology in overweight and obese orthodontic adolescent patients. J Pre-Clin Clin Res. 2017;11(1):42-5.

6. Sanchez-Perez L, Irigoyen ME, Zepeda M. Dental caries, tooth eruption timing and obesity: a longitudinal study in a group of Mexican schoolchildren. Acta Odontol Scand. 2010;68(1):57-64. doi: 10.26444/jpccr/75138

7. Hong L, Ahmed A, McCunniff M, Overman P, Mathew M. Obesity and dental caries in children aged 2-6 years in the United States: National Health and Nutrition Examination Survey 1999-2002. J Public Health Dent. 2008;68(4):227-33. doi: 10.1111/j.17527325.2008.00083.x

8. Kline L, Jones-Smith J, Jaime Miranda J, Pratt M, Reis RS, Rivera JA, et al. A research agenda to guide progress on childhood obesity prevention in Latin America. Obes Rev. 2017;18 Suppl 2:19-27. doi: 10.1111/obr.12572

9. Mazur A, Caroli M, Radziewicz-Winnicki I, Nowicka P, Weghuber D, Neubauer D, et al. Reviewing and addressing the link between mass media and the increase in obesity among European children: The European Academy of Paediatrics (EAP) and The European Childhood Obesity Group (ECOG) consensus statement. Acta Paediatr. 2018;107(4):568-76. doi: 10.1111/apa.14136 